\title{
Exponentially Stabilizing Controllers for Multi-Contact 3D Bipedal Locomotion*
}

\author{
Kaveh Akbari Hamed ${ }^{1}$, Robert D. Gregg ${ }^{2}$, and Aaron D. Ames ${ }^{3}$
}

\begin{abstract}
Models of bipedal walking are hybrid with continuous-time phases representing the Lagrangian stance dynamics and discrete-time transitions representing the impact of the swing leg with the walking surface. The design of continuous-time feedback controllers that exponentially stabilize periodic gaits for hybrid models of underactuated 3D bipedal walking is a significant challenge. We recently introduced a method based on an iterative sequence of optimization problems involving bilinear matrix inequalities (BMIs) to systematically design stabilizing continuous-time controllers for single domain hybrid models of underactuated bipedal robots with point feet. This paper addresses the exponential stabilization problem for multi-contact walking gaits with nontrivial feet. A family of parameterized continuous-time controllers is proposed for different phases of the walking cycle. The BMI algorithm is extended to the multi-domain hybrid models of anthropomorphic 3D walking locomotion to look for stabilizing controller parameters. The Poincaré map is addressed and a new set of sufficient conditions is presented that guarantees the convergence of the BMI algorithm to a stabilizing set of controller parameters at a finite number of iterations. The power of the algorithm is ultimately demonstrated through the design of stabilizing virtual constraint controllers for dynamic walking of a $3 \mathrm{D}$ humanoid model with 28 state variables and 275 controller parameters.
\end{abstract}

\section{INTRODUCTION}

This paper presents a systematic algorithm to design continuous-time controllers that exponentially stabilize periodic gaits for multi-domain hybrid dynamical systems describing anthropomorphic 3D bipedal walking with nontrivial feet. The hybrid systems consist of distinct phases (or domains) with the dynamic model in each phase depending on the nature of the contact between the feet and the ground. We consider a family of parameterized continuous-time feedback controllers that preserve a desired periodic walking gait for the closed-loop system. The algorithm is developed based on the Poincaré return map and employs an iterative sequence of optimization problems involving bilinear matrix inequalities (BMIs) to search for stabilizing controller parameters. The

*This material is based upon work supported by the National Science Foundation under Grant Number 1637704. The content is solely the responsibility of the authors and does not necessarily represent the official views of the NSF. R. D. Gregg holds a Career Award at the Scientific Interface from the Burroughs Wellcome Fund.

${ }^{1} \mathrm{~K}$. Akbari Hamed is with the Department of Mechanical Engineering, San Diego State University, San Diego, CA 92182-1323 USA kakbarihamed@mail.sdsu.edu

${ }^{2} \mathrm{R}$. D. Gregg is with the Departments of Bioengineering and Mechanical Engineering, University of Texas at Dallas, Richardson, TX 75080-3021 USA rgreggeutdallas.edu

${ }^{3}$ A. D. Ames is with the Department of Mechanical and Civil Engineering, California Institute of Technology, Pasadena, CA 91125 USA ames@cds.caltech.edu framework can ameliorate specific challenges in the design of stabilizing controllers for bipedal robots arising from high dimensionality and underactuation. The power of the algorithm is ultimately demonstrated through the design of a set of nonlinear feedback controllers with 275 controller parameters for dynamic walking of a 3D humanoid model with 28 state variables.

The control method most widely used on humanoid robots is based on a heuristic notion of gait stability, known as the zero moment point (ZMP) criterion [1], [2]. The ZMP criterion assumes full actuation and results in quasi-static and flat-footed walking. The ZMP assumption required for flat-footed walking is not satisfied during underactuated phases of human walking, e.g., initial heel contact and final toe-off. Feedback control algorithms that directly deal with the hybrid nature of legged locomotion have come out of controlled symmetries [3], hybrid reduction [4], [5], transverse linearization [6], and hybrid zero dynamics (HZD) [7], [8]. Controlled symmetries and hybrid reduction typically assume full actuation (though in specific cases one degree of underactuation can be achieved [9]). However, transverse linearization and HZD-based controllers are the only controllers of these methods that explicitly deal with general cases of underactuation. HZD-based controllers have been validated numerically and experimentally for $2 \mathrm{D}$ and $3 \mathrm{D}$ bipedal robots [10]-[19], 2D and 3D powered prosthetic legs [20]-[25], exoskeletons [26], monopedal [27] and quadruped robots [28]. In this approach, a set of output functions, referred to as virtual constraints, is defined for the continuous-time dynamics of the system and asymptotically driven to zero by the input-output (I-O) linearizing feedback controller [29]. For mechanical systems with more than one degree of underactuation, the stability of walking gaits depends on the choice of the virtual constraints [30], [31]. Anthropomorphic heel-to-toe walking has been achieved in planar models [32, Chapter 10] and most recently in a 3D robot [18] using the HZD controllers. In particular, [18] presented an efficient optimization framework (motion planning) to generate HZD gaits. Although the gaits of [18] are stable, there is currently no systematic algorithm to choose asymptotically stabilizing virtual constraints for a given 3D walking gait. We are trying to answer this fundamental question: how to present an effective optimization algorithm to choose stabilizing virtual constraints for a generated 3D gait with high degrees of underactuation?

The most basic tool to investigate the stability of periodic orbits for hybrid dynamical systems is the method of Poincaré sections [8], [33]-[36] that describes the evolution 
of the system on a hyperplane transversal to the orbit by a discrete-time system, referred to as the Poincaré return map. One drawback of Poincaré sections analysis is the lack of a closed-form expression for the Poincaré map and its Jacobian linearization, which complicates the design of continuoustime feedback controllers for hybrid models of bipedal walking. To tackle this roadblock, we have recently proposed an algorithm based on an iterative sequence of optimization problems involving BMIs to choose stabilizing continuoustime controllers from a family of parameterized feedback laws [30], [37], [38]. These controllers were numerically and experimentally verified for single domain hybrid models of bipedal robots with point feet [39], [40].

The current paper addresses the important problem of designing stabilizing virtual constraint controllers to achieve dynamic and anthropomorphic multi-contact walking. In particular, the contributions of the paper are as follows: 1) A multi-domain hybrid system formulation for 3D human walking is first presented that includes the "heel-roll", "flatfooted", and "toe-roll" phases (see Fig. 1); 2) A family of parameterized nonlinear feedback controllers including parameterized virtual constraint controllers is presented for different phases of the walking cycle; 3) The exponential stabilization problem and Poincaré map for the multi-domain hybrid models of walking are investigated; 4) The BMI algorithm is extended to the models of anthropomorphic bipedal walking; 5) A new set of sufficient conditions are presented to guarantee the convergence of the algorithm to a stabilizing set of controller parameters at a finite number of iterations; and 6) Finally, the theoretical framework is numerically confirmed through designing stabilizing virtual constraint controllers with 275 controller parameters for dynamic walking of a 3D humanoid model with 28 state variables. Unlike the sufficient conditions in [37] that require extensive computational techniques based on the third-order derivative of the Poincare map, the new conditions only depend on the first- and second-order derivatives of the Poincaré map and thereby can be effectively verified.

\section{HYBRID MODEL OF WALKING}

We consider a multi-domain hybrid system formulation to describe 3D and anthropomorphic walking on a flat surface. The hybrid model of walking is assumed to consist of three distinct single support phases $\{1,2,3\}$ executed in a fixed order, i.e., they define the cyclic graph $1 \rightarrow 2 \rightarrow 3 \rightarrow 1$ (see Fig. 1). The walking cycle starts with an underactuated phase in which the stance heel acts as a pivot point on the ground (i.e., Phase 1 or "heel-roll"), followed by a fully actuated phase in which the stance foot remains flat on the walking surface (i.e., Phase 2 or "flat-footed" phase), and finishes with an alternative underactuated phase where the stance toe is in contact with the ground and the stance foot rotates about the toe (i.e., Phase 3 or "toe-roll"). The double support phase is assumed to be instantaneous and occurs at the end of Phase 3 (i.e., discrete transition $3 \rightarrow 1$ ). The transition from the heel-roll to flat-footed phase (i.e., $1 \rightarrow 2$ ) happens when the stance toe contacts the walking surface. In an analogous

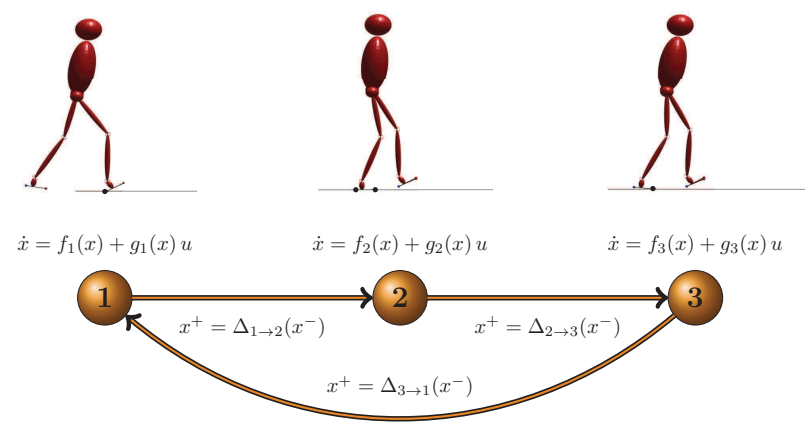

Fig. 1: Illustration of the multi-domain hybrid model of 3D walking. Continuous-time phases and discrete-time transitions are represented by the vertices and edges of the directed cycle, respectively. The black dots indicate the constraints enforced in each continuous-time phase. The hybrid model consists of two underactuated phases, shown by the vertices 1 (heel-roll) and 3 (toe-roll). The vertex 2 (flat-footed) is assumed to be fully-actuated.

manner, the transition from the toe-roll to heel-roll phase (i.e., $3 \rightarrow 1$ ) occurs when the swing heel impacts the ground. Finally, the discrete transition from the flat-footed to toeroll phase (i.e., $2 \rightarrow 3$ ) is initiated by causing the stance heel to take-off the walking surface. In our formulation, the continuous-time phases are represented by the vertices of the graph shown by $i \in \mathcal{I}:=\{1,2,3\}$, whereas the discrete-time transitions are denoted by the edges shown by $e \in \mathcal{E}:=\{1 \rightarrow 2,2 \rightarrow 3,3 \rightarrow 1\}$. For later purposes, we define the index of the next phase function as $\mu: \mathcal{I} \rightarrow \mathcal{I}$ by $\mu(i):=\bmod (i, 3)+1$, in which "mod" represents the modulo operation.

We consider a common state vector to derive equations of motion during all phases of walking often termed the "unpinned" model of the robot. This vector, shown by $x:=$ $\left(q^{\top}, \dot{q}^{\top}\right)^{\top}$, represents the state variables for the mechanical system during the flight phase (i.e., no leg in contact with the ground). Here, $q \in \mathcal{Q} \subset \mathbb{R}^{n_{q}}$ and $\dot{q} \in \mathbb{R}^{n_{q}}$ denote the generalized position and velocity vectors, respectively, for some positive integer $n_{q}$. It is assumed that $q$ consists of three components for the translational motion of the robot, three components for the absolute orientation of the system with respect to a world frame, and a set of internal configuration variables to describe the shape of the robot. The configuration space is also represented by an open and connected set $\mathcal{Q}$. We remark that the number of degrees of freedom (DOFs) for each phase depends on the foot-ground contact nature. The holonomic constraints arising from different contact conditions will be considered in Sections II-A and II-B to extract the equations of motions.

The open-loop hybrid control system representing multicontact walking is given by the following tuple (see Fig. 1)

$$
\mathscr{H} \mathscr{L}^{\mathrm{ol}}=(\Lambda, \mathcal{X}, \mathcal{U}, \mathcal{D}, \mathcal{S}, \Delta, F G),
$$

where

- $\Lambda=(\mathcal{I}, \mathcal{E})$ is a directed cycle with vertices $\mathcal{I}=$ $\{1,2,3\}$ and edges $\mathcal{E}=\left\{e_{i}=\{i \rightarrow \mu(i)\}\right\}_{i \in \mathcal{I}}$; 
- $\mathcal{X}:=\left\{\mathcal{X}_{i}\right\}_{i \in \mathcal{I}}$ is a set of state manifolds, in which $\mathcal{X}_{i} \subset \mathbb{R}^{2 n_{q}}$ denotes the state manifold for the vertex $i \in \mathcal{I}$

- $\mathcal{U}:=\left\{\mathcal{U}_{i}\right\}_{i \in \mathcal{I}}$ is a set of admissible controls, in which $\mathcal{U}_{i} \subset \mathbb{R}^{m}$ represents the set of admissible control inputs for the vertex $i \in \mathcal{I}$ and some positive integer $m$;

- $\mathcal{D}:=\left\{\mathcal{D}_{i}\right\}_{i \in \mathcal{I}}$ is a set of domains of admissibility, in which $\mathcal{D}_{i} \subset \mathcal{X}_{i} \times \mathcal{U}_{i}$ is a smooth submanifold of $\mathbb{R}^{2 n_{q}} \times$ $\mathbb{R}^{m}$ for $i \in \mathcal{I}$;

- $\mathcal{S}:=\left\{\mathcal{S}_{e}\right\}_{e \in \mathcal{E}}$ is a set of guards, in which $\mathcal{S}_{i \rightarrow \mu(i)} \subset \mathcal{D}_{i}$ for $i \in \mathcal{I}$

- $\Delta:=\left\{\Delta_{e}\right\}_{e \in \mathcal{E}}$ is a set of reset laws, where $\Delta_{i \rightarrow \mu(i)}$ is a smooth discrete-time system represented by $x^{+}=$ $\Delta_{i \rightarrow \mu(i)}\left(x^{-}\right)$for $i \in \mathcal{I}$, and $x^{-}(t):=\lim _{\tau \nearrow_{t}} x(\tau)$ and $x^{+}(t):=\lim _{\tau \searrow t} x(\tau)$ denote the left and right limits of the state trajectory $x(t)$, respectively; and

- $F G:=\left\{\left(f_{i}, g_{i}\right)\right\}_{i \in \mathcal{I}}$, where $\left(f_{i}, g_{i}\right)$ is a control system on $\mathcal{D}_{i}$, i.e., $\dot{x}=f_{i}(x)+g_{i}(x) u$ for $(x, u) \in \mathcal{D}_{i}$ and $i \in \mathcal{I}$ with $f_{i}$ and columns of the $g_{i}$ matrix being smooth on $\mathcal{X}_{i}$.

\section{A. Continuous-Time Phases}

This section addresses the continuous-time dynamics of the bipedal walker during different phases of walking. Throughout this paper, we shall assume that the feet are "forward facing" with one "heel" point (i.e., back end) and two "toe" points (i.e., forward ends), referred to as the right and left toes. In particular, the biped walker's foot prints form a triangular on the walking surface whose vertices are denoted by the heel ("h"), right toe ("rt"), and left toe ("lt"). We represent the Cartesian coordinates of these vertices by $p_{j}^{l} \in \mathbb{R}^{3}$, where the subscript $j \in\{$ st, sw $\}$ denotes the stance and swing feet, and the superscript $l \in\{\mathrm{h}, \mathrm{rt}, \mathrm{lt}\}$ represents the vertex type. We further assume that a coordinate frame has been rigidly attached to the stance foot. The orientation of this frame with respect to an inertial world frame is given by the rotation matrix $R_{\mathrm{st}}(q) \in \mathrm{SO}(3)$, where $\mathrm{SO}(3)$ is the rotation group in $\mathbb{R}^{3}$. During the continuous-time phase $i \in \mathcal{I}$, the evolution of the mechanical system is described by the following set of ordinary differential equations (ODEs) [41]:

$$
\begin{aligned}
& D(q) \ddot{q}+C(q, \dot{q}) \dot{q}+G(q)=B u+J_{i}^{\top}(q) \lambda_{i} \\
& J_{i}(q) \ddot{q}+\frac{\partial}{\partial q}\left(\frac{\partial J_{i}}{\partial q}(q) \dot{q}\right) \dot{q}=0 .
\end{aligned}
$$

In (2), $D(q) \in \mathbb{R}^{n_{q} \times n_{q}}$ denotes the positive-definite massinertia matrix, $C(q, \dot{q}) \dot{q} \in \mathbb{R}^{n_{q}}$ represents the Coriolis and centrifugal terms, $G(q) \in \mathbb{R}^{n_{q}}$ denotes the gravitational terms, and $B \in \mathbb{R}^{n_{q} \times m}$ represents the input distribution matrix with the property $\operatorname{rank}(B)=m$. Moreover, $J_{i}(q)$ and $\lambda_{i}$ represent the corresponding contact Jacobian matrix and Lagrange multipliers, respectively. One can eliminate the Lagrange multipliers $\lambda_{i}$ and solve the set of ODEs (2) and (3) for $\ddot{q}$ as follows

$$
D(q) \ddot{q}+F_{i}(q, \dot{q})=T_{i}(q) u,
$$

where $T_{i}:=\Pi_{i} B, \Pi_{i}:=I-J_{i}^{\top}\left(J_{i} D^{-1} J_{i}^{\top}\right)^{-1} J_{i} D^{-1}$, and $F_{i}:=\Pi_{i}(C \dot{q}+G)+J_{i}^{\top}\left(J_{i} D^{-1} J_{i}^{\top}\right)^{-1} \frac{\partial}{\partial q}\left(\frac{\partial J_{i}}{\partial q} \dot{q}\right) \dot{q}$ We remark that (4) can be easily expressed in the input-affine format $\dot{x}=f_{i}(x)+g_{i}(x) u$ for all $i \in \mathcal{I}$. Furthermore, the state manifolds $\mathcal{X}_{i}$ are given by

$$
\begin{aligned}
& \mathcal{X}_{1}:=\left\{\left(q^{\top}, \dot{q}^{\top}\right)^{\top} \in \mathrm{T} \mathcal{Q} \mid p_{\mathrm{st}}^{\mathrm{h}}(q)=\text { const, } J_{1}(q) \dot{q}=0\right\} \\
& \mathcal{X}_{2}:=\left\{\left(q^{\top}, \dot{q}^{\top}\right)^{\top} \in \mathrm{T} \mathcal{Q} \mid p_{\mathrm{st}}^{\mathrm{h}}(q)=\mathrm{const}, R_{\mathrm{st}}(q)=\mathrm{const},\right. \\
&\left.J_{2}(q) \dot{q}=0\right\} \\
& \mathcal{X}_{3}:=\left\{\left(q^{\top}, \dot{q}^{\top}\right)^{\top} \in \mathrm{T} \mathcal{Q} \mid p_{\mathrm{st}}^{\mathrm{rt}}(q)=\mathrm{const}, p_{\mathrm{st}}^{\mathrm{lt}}(q)=\mathrm{const},\right. \\
&\left.J_{3}(q) \dot{q}=0\right\},
\end{aligned}
$$

where $\mathrm{T} \mathcal{Q}:=\mathcal{Q} \times \mathbb{R}^{n_{q}}$ represents the tangent bundle of $\mathcal{Q}$ and "const" stands for the constant. We can show that 1) $\operatorname{dim}\left(\mathcal{X}_{1}\right)=2\left(n_{q}-3\right), \operatorname{dim}\left(\mathcal{X}_{2}\right)=2\left(n_{q}-6\right), \operatorname{dim}\left(\mathcal{X}_{3}\right)=$ $2\left(n_{q}-5\right)$, and 2$)$ Phase 2 is fully-actuated if and only if $n_{q}=m+6$. Finally, one can present a set of domains of admissibility $\left\{\mathcal{D}_{i}\right\}_{i \in \mathcal{I}}$ to represent the unilateral constraints on the forces and moments at the ground-foot interface similar to [42].

\section{B. Discrete-Time Transitions}

This section addresses the discrete transitions for the hybrid model of walking (1). The transitions $1 \rightarrow 2$ and $3 \rightarrow 1$ can be represented by instantaneous and inelastic impact models for which the ground reaction forces/moments at the contacting points are impulsive [43]. In particular, integration of the flight phase dynamics subject to the impulsive forces/moments over the infinitesimal impact interval yields

$$
\begin{aligned}
& D(q)\left(\dot{q}^{+}-\dot{q}^{-}\right)=J_{i \rightarrow \mu(i)}^{\top}(q) \lambda_{i \rightarrow \mu(i)} \\
& J_{i \rightarrow \mu(i)}(q) \dot{q}^{+}=0,
\end{aligned}
$$

where $i \in \mathcal{I} \backslash\{2\}, J_{i \rightarrow \mu(i)}(q)$ is the corresponding impact Jacobian matrix, $\lambda_{i \rightarrow \mu(i)}$ represents the intensity of the impulsive forces/moments, and $\dot{q}^{-}$and $\dot{q}^{+}$denote the generalized velocity components right before and after the impact event, respectively. The transition from the flat-footed to toe-roll phase (i.e., $2 \rightarrow 3$ ) finally assumes the continuity of position and velocity components, and consequently, $\Delta_{2 \rightarrow 3}(x):=$ $\mathrm{id}_{\mathrm{T} \mathcal{Q}}$, where $\mathrm{id}_{\mathrm{T} \mathcal{Q}}$ represents the identity function on $\mathrm{T} \mathcal{Q}$.

\section{FAMILY OF PARAMETERIZED CONTINUOUS-TIME FEEDBACK CONTROLLERS}

The objective of this section is to present a family of parameterized and smooth continuous-time feedback controllers that exponentially stabilize periodic gaits for the multi-domain hybrid model of walking (1).

\section{A. Periodic Walking Gaits and Phasing Variables}

Throughout this paper, we shall assume that there is a period-one orbit for the open-loop hybrid model (1) that is transversal to the guards. In particular, we make the following assumption. 
Assumption 1 (Transversal Period-One Walking Gait): There exist 1) finite numbers $\left.T_{i}^{\star}>0,2\right)$ smooth nominal control inputs $u_{i}:\left[0, T_{i}^{\star}\right] \rightarrow \mathcal{U}$, and 3) smooth nominal state trajectories $\varphi_{i}^{\star}:\left[0, T_{i}^{\star}\right] \rightarrow \mathcal{X}_{i}$ for $i \in \mathcal{I}$ such that

1) the continuous-time ODEs are satisfied, i.e., $\dot{\varphi}_{i}^{\star}(t)=$ $f_{i}\left(\varphi_{i}^{\star}(t)\right)+g_{i}\left(\varphi_{i}^{\star}(t)\right) u_{i}^{\star}(t)$ for all $t \in\left[0, T_{i}^{\star}\right]$ and $i \in \mathcal{I}$ with the properties a) $\left(\varphi_{i}^{\star}(t), u_{i}^{\star}(t)\right) \notin \mathcal{S}_{i \rightarrow \mu(i)}$ for $t \in\left[0, T_{i}^{\star}\right)$ and b) $\left(\varphi_{i}^{\star}\left(T_{i}^{\star}\right), u_{i}^{\star}\left(T_{i}^{\star}\right)\right) \in \mathcal{S}_{i \rightarrow \mu(i)}$;

2) the periodicity condition is met, i.e., $\varphi_{\mu(i)}^{\star}(0)=$ $\Delta_{i \rightarrow \mu(i)}\left(\varphi_{i}^{\star}\left(T_{i}^{\star}\right)\right), i \in \mathcal{I}$; and

$3)$ the transversality conditions hold in the sense that the nominal state trajectories are transversal to the corresponding guards.

Then, $\mathcal{O}:=\mathcal{O}_{1} \cup \mathcal{O}_{2} \cup \mathcal{O}_{3}$ is a period-one gait for the openloop hybrid model (1) that is transversal to the guards. In our notation, $\mathcal{O}_{i}:=\left\{x=\varphi_{i}^{\star}(t) \mid 0 \leq t<T_{i}^{\star}\right\}$ is the projection of the orbit onto the state manifold $\mathcal{X}_{i}$ for $i \in \mathcal{I}$. For later purposes, we define $\left\{x_{i}^{\star}\right\}$ as the intersection of $\overline{\mathcal{O}}_{i}$ and the guard $\mathcal{S}_{i \rightarrow \mu(i)}$, where $\overline{\mathcal{O}}_{i}$ denotes the set closure of the orbit $\mathcal{O}_{i}$.

We shall consider $\mathcal{O}$ as the desired orbit to be exponentially stabilized. In order to develop time-invariant feedback controllers, we make use of the concept of a phasing variable.

Assumption 2 (Phasing Variables): There exist smooth and real-valued functions $\theta_{i}: \mathcal{X}_{i} \rightarrow \mathbb{R}, i \in \mathcal{I}$, referred to as the phasing variables, that are strictly increasing functions of time on $\overline{\mathcal{O}}_{i}$, that is, $\dot{\theta}_{i}(x)>0$ for all $x \in \overline{\mathcal{O}}_{i}$.

The phasing variable is key to obtaining time-invariant feedback controllers. It represents the progression of the robot on the walking gait and plays the role of time in time-invariant feedback controllers. Reference [44] shows that the existence of phasing variables follows directly from Assumption 1.

\section{B. Family of Parameterized Controllers}

We consider a family of parameterized state feedback controllers as follows:

$$
u=\Gamma_{i}\left(x, \xi_{i}\right), \quad i \in \mathcal{I}
$$

for the continuous-time phases of the hybrid model of walking, in which $\Gamma_{i}: \mathcal{X}_{i} \times \Xi_{i} \rightarrow \mathcal{U}_{i}$ is a $\mathcal{C}^{\infty}$ feedback law parameterized by a set of adjustable controller parameters $\xi_{i} \in \Xi_{i}$ to be determined. In our notation, $\Xi_{i} \subset \mathbb{R}^{p_{i}}$ denotes the set of admissible controller parameters for some positive integer $p_{i}$. Throughout this paper, we shall assume that the family of feedback controllers (7) satisfies the following invariance condition.

Assumption 3 (Invariant Periodic Orbit): The orbit $\overline{\mathcal{O}}_{i}$ is invariant under the change of the controller parameters $\xi_{i} \in \Xi_{i}$, that is, $\Gamma_{i}\left(\varphi_{i}^{\star}(t), \xi_{i}\right)=u_{i}^{\star}(t)$, for all $t \in\left[0, T_{i}^{\star}\right]$ and $i \in \mathcal{I}$. In particular, $\overline{\mathcal{O}}_{i}$ is an integral curve of the parameterized and closed-loop ODE $\dot{x}=f_{i}^{\mathrm{cl}}\left(x, \xi_{i}\right):=$ $f_{i}(x)+g_{i}(x) \Gamma_{i}\left(x, \xi_{i}\right)$ for all $\xi_{i} \in \Xi_{i}$.

Assumption 3 will allow the BMI algorithm of Section IV-B to look for stabilizing controller parameters while preserving the desired orbit for the closed-loop hybrid system.

\section{Input-Output Linearizing Feedback Controllers}

This section presents an important family of parameterized state feedback controllers (7), referred to as virtual constraint controllers, that satisfies the invariance condition in Assumption 3. Virtual constraints are defined as holonomic output functions $y(x)$ for continuous-time portions of hybrid models of walking to coordinate the links of bipedal robots within a stride [7], [10], [11], [13], [20], [21], [30], [33], [45], [46]. In this paper, we consider a parameterized family of virtual constraints for continuous-time phases as follows:

$$
y_{i}\left(x, \xi_{i}\right)=H_{i}\left(\xi_{i}\right)\left(q-q_{\mathrm{d}, i}\left(\theta_{i}\right)\right),
$$

where $H_{i}\left(\xi_{i}\right)$ is the output matrix to be determined and parameterized by $\xi_{i}$, and $q_{\mathrm{d}, i}\left(\theta_{i}\right)$ represents the desired evolution of the configuration variables on the orbit $\overline{\mathcal{O}}_{i}$ in terms of the phasing variable $\theta_{i}$. In particular, $q_{\mathrm{d}, i}\left(\theta_{i}\right):=$ $\pi_{q}\left(x_{\mathrm{d}, i}\left(\theta_{i}\right)\right)$ and $x_{\mathrm{d}, i}\left(\theta_{i}\right):=\left.\varphi_{i}^{\star}(t)\right|_{t=\left(\theta_{i}^{\star}\right)^{-1}\left(\theta_{i}\right)}$, where $\theta_{i}=\theta_{i}^{\star}(t)$ and $t=\left(\theta_{i}^{\star}\right)^{-1}\left(\theta_{i}\right)$ denote the time profile of the phasing variable $\theta_{i}$ on the orbit $\overline{\mathcal{O}}_{i}$ and its inverse function, respectively. Furthermore, $\pi_{q}(q, \dot{q}):=q$ represents the canonical projection onto the configuration space.

During the heel- and toe-roll phases, the mechanical system is underactuated and the output functions are chosen to have the same dimension as the control input, that is, $\operatorname{dim}\left(y_{1}\right)=\operatorname{dim}\left(y_{3}\right)=\operatorname{dim}(u)=m$ (i.e., $H_{1} \in \mathbb{R}^{m \times n_{q}}$ and $H_{3} \in \mathbb{R}^{m \times n_{q}}$ ). The I-O linearizing controllers are then employed to zero the output functions $y_{1}$ and $y_{3}$ as follows $\Gamma_{i}\left(x, \xi_{i}\right)=-\left(\mathrm{L}_{g_{i}} \mathrm{~L}_{f_{i}} y_{i}\right)^{-1}\left(\mathrm{~L}_{f_{i}}^{2} y_{i}+\frac{K_{D}}{\epsilon} \mathrm{L}_{f_{i}} y_{i}+\frac{K_{P}}{\epsilon^{2}} y_{i}\right)$,

which results in the output dynamics $\ddot{y}_{i}+\frac{K_{D}}{\epsilon} \dot{y}_{i}+\frac{K_{P}}{\epsilon^{2}} y_{i}=0$. Here, we have assumed that the output function $y_{i}\left(x, \xi_{i}\right)$ has relative degree vector $(2,2, \cdots, 2)$ with respect to $u$ on an open neighborhood of $\mathcal{O}_{i}$ for $i \in \mathcal{I} \backslash\{2\}$. In addition, $K_{P}>0$ and $K_{D}>0$ are positive PD gains, and $\epsilon>0$ is a scalar to tune the settling time. We remark that the feedback controller (9) renders the corresponding parameterized zero dynamics manifold $\mathcal{Z}_{i}\left(\xi_{i}\right)$ forward invariant under the flow of the closed-loop ODE $\dot{x}=f_{i}^{\text {cl }}\left(x, \xi_{i}\right)$, in which $\mathcal{Z}_{i}\left(\xi_{i}\right):=$ $\left\{x \in \mathcal{X}_{i} \mid y_{i}\left(x, \xi_{i}\right)=\mathrm{L}_{f_{i}} y_{i}\left(x, \xi_{i}\right)=0\right\}$.

During the flat-footed phase, the system is assumed to be fully actuated and in order to have a nontrivial zero dynamics manifold, we consider an $(m-1)$-dimensional output function to be regulated, i.e., $\operatorname{dim}\left(y_{2}\right)=\operatorname{dim}(u)-1=$ $m-1$ or equivalently $H_{2} \in \mathbb{R}^{(m-1) \times n_{q}}$. To zero the output function $y_{2}$, we then split the control input as follows

$$
u=\left(u_{\mathrm{rem}}^{\top}, u_{\mathrm{A}}\right)^{\top}
$$

where $u_{\mathrm{A}} \in \mathbb{R}$ and $u_{\mathrm{rem}} \in \mathbb{R}^{m-1}$ denote the torque at the sagittal stance ankle joint and torques at the remaining actuated joints, respectively. Differentiating the output function $y_{2}$ along the solutions of the closed-loop system yields

$$
\ddot{y}_{2}=\mathrm{L}_{f_{2}}^{2} y_{2}+\mathrm{L}_{g_{2}^{\text {rem }}} \mathrm{L}_{f_{2}} y_{2} u_{\mathrm{rem}}+\mathrm{L}_{g_{2}^{\mathrm{A}}} \mathrm{L}_{f_{2}} y_{2} u_{\mathrm{A}}
$$

in which $g_{2}(x)=\left[g_{2}^{\mathrm{rem}}(x) g_{2}^{\mathrm{A}}(x)\right]$ is a partitioning of the $g_{2}$ matrix corresponding to (10). The I-O linearizing controller 
is then chosen as

$$
\begin{aligned}
u_{\mathrm{rem}}\left(x, \xi_{2}, u_{\mathrm{A}}\right)= & -\left(\mathrm{L}_{g_{2}^{\mathrm{rem}}} \mathrm{L}_{f_{2}} y_{2}\right)^{-1}\left(\mathrm{~L}_{f_{2}}^{2} y_{2}+\mathrm{L}_{g_{2}^{\mathrm{A}}} \mathrm{L}_{f_{2}} y_{2} u_{\mathrm{A}}\right. \\
& \left.+\frac{K_{D}}{\epsilon} \mathrm{L}_{f_{2}} y_{2}+\frac{K_{P}}{\epsilon^{2}} y_{2}\right)
\end{aligned}
$$

that renders the corresponding zero dynamics manifold (i.e., $\mathcal{Z}_{2}\left(\xi_{2}\right)$ ) forward invariant under the flow of the closed-loop system regardless of the choice of $u_{\mathrm{A}}$. In order to preserve the orbit $\mathcal{O}_{2}$ for the system, we can employ the following LQR controller for the sagittal stance ankle torque

$$
u_{\mathrm{A}}\left(x, \xi_{2}\right)=u_{\mathrm{A}}^{\star}\left(\theta_{2}\right)-K_{2}\left(\xi_{2}\right)\left(x-x_{\mathrm{d}, 2}\left(\theta_{2}\right)\right),
$$

where $u_{\mathrm{A}}^{\star}\left(\theta_{2}\right)$ represents the nominal stance ankle torque on the desired orbit in terms of the phasing variable $\theta_{2}$, i.e., $u_{\mathrm{A}}^{\star}\left(\theta_{2}\right):=\left.\pi_{u} \circ u_{2}^{\star}(t)\right|_{t=\left(\theta_{2}^{\star}\right)^{-1}\left(\theta_{2}\right)}, \pi_{u}\left(u_{\mathrm{rem}}, u_{\mathrm{A}}\right):=u_{\mathrm{A}}$ is the canonical projection map onto the stance ankle torque, $x_{\mathrm{d}, 2}\left(\theta_{2}\right)$ denotes the desired state variables on the orbit, and $K_{2}\left(\xi_{2}\right)$ is the stance ankle controller gain to be determined and parameterized by $\xi_{2}$. The state feedback controller for the flat-footed phase is finally chosen as $\Gamma_{2}\left(x, \xi_{2}\right)=$ $\left(u_{\text {rem }}^{\top}, u_{\mathrm{A}}\right)^{\top}$. According to the construction procedure, $\mathcal{O}_{i} \subset$ $\mathcal{Z}_{i}\left(\xi_{i}\right)$ for all $\xi_{i} \in \Xi_{i}$ and $i \in \mathcal{I}$. Furthermore from [30, Example 2], the I-O linearizing feedback controllers satisfy the invariance condition, i.e., $\frac{\partial \Gamma_{i}}{\partial x}\left(x, \xi_{i}\right)=0$ for all $x \in \overline{\mathcal{O}}_{i}$ and $i \in \mathcal{I}$. Without loss of generality, we can assume that $\xi_{i}=\operatorname{vec}\left(H_{i}\right)$ for $i \in \mathcal{I} \backslash\{2\}$ and $\xi_{2}=\left(\operatorname{vec}\left(H_{2}\right)^{\top}, K_{2}\right)^{\top}$, in which "vec" represents the vectorization operator.

\section{EXPONENTIAL STABILIZATION}

This section addresses the exponential stabilization problem. The objective is to systematically tune the controller parameters $\xi:=\left(\xi_{1}^{\top}, \xi_{2}^{\top}, \xi_{3}^{\top}\right)^{\top} \in \Xi$ such that the periodic orbit $\mathcal{O}$ becomes orbitally exponentially stable for the closedloop hybrid system, where $\Xi:=\Xi_{1} \times \Xi_{2} \times \Xi_{3} \in \mathbb{R}^{p}$ and $p:=p_{1}+p_{2}+p_{3}$.

\section{A. Poincaré Return Map}

We make use of the Poincaré sections approach to study the stabilization problem. For this goal, let us denote the unique solution of the parameterized and closed-loop ODE $\dot{x}=f_{i}^{\text {cl }}\left(x, \xi_{i}\right), i \in \mathcal{I}$ with the initial condition $x(0)$ by $x(t)=\varphi_{i}\left(t, x(0), \xi_{i}\right)$ for all $t \geq 0$ in the maximal interval of existence. Since the family of control inputs has been specified, one can present a set of guards $\left\{\hat{\mathcal{S}}_{i \rightarrow \mu(i)}\right\}_{i \in \mathcal{I}} \subset \mathcal{X}$ for the closed-loop hybrid model of walking on which the discrete transitions $i \rightarrow \mu(i)$ occur. The time-to-switching function is defined as $T_{i}: \mathcal{X}_{i} \times \Xi_{i} \rightarrow \mathbb{R}_{>0}, i \in \mathcal{I}$ by

$$
T_{i}\left(x(0), \xi_{i}\right):=\inf \left\{t>0 \mid \varphi_{i}\left(t, x(0), \xi_{i}\right) \in \hat{\mathcal{S}}_{i \rightarrow \mu(i)}\right\}
$$

as the first time at which the solution $\varphi_{i}\left(t, x(0), \xi_{i}\right)$ intersects the switching surface $\hat{\mathcal{S}}_{i \rightarrow \mu(i)}$. We then define the flow of Phase $i$ as $P_{i}: \hat{\mathcal{S}}_{\mu^{-1}(i) \rightarrow i} \times \Xi_{i} \rightarrow \hat{\mathcal{S}}_{i \rightarrow \mu(i)}$ by

$$
\begin{aligned}
& P_{i}\left(x, \xi_{i}\right) \\
& \quad:=\varphi_{i}\left(T_{i}\left(\Delta_{\mu^{-1}(i) \rightarrow i}(x), \xi_{i}\right), \Delta_{\mu^{-1}(i) \rightarrow i}(x), \xi_{i}\right),
\end{aligned}
$$

where $\mu^{-1}($.$) represents the inverse of the next phase func-$ tion $\mu($.$) . The Poincaré return map is finally defined as$ $P: \hat{\mathcal{S}}_{1 \rightarrow 2} \times \Xi \rightarrow \hat{\mathcal{S}}_{1 \rightarrow 2}$ by the following composition rule

$$
P(x, \xi):=P_{1}\left(P_{3}\left(P_{2}\left(x, \xi_{2}\right), \xi_{3}\right), \xi_{1}\right)
$$

that describes the evolution of the mechanical system on the Poincaré section $\hat{\mathcal{S}}_{1 \rightarrow 2}$ according to the following discretetime system

$$
x[k+1]=P(x[k], \xi), \quad k=0,1, \cdots .
$$

From the construction procedure and the invariance condition, $x_{1}^{\star}$ is an invariant fixed-point of the Poincaré map $P$, that is, $P\left(x_{1}^{\star}, \xi\right)=x_{1}^{\star}$ for all $\xi \in \Xi$. Linearization of (15) around the fixed point $x_{1}^{\star}$ then yields ${ }^{1}$

$$
\delta x[k+1]=\frac{\partial P}{\partial x}\left(x_{1}^{\star}, \xi\right) \delta x[k],
$$

where $\delta x[k]:=x[k]-x_{1}^{\star}$.

Problem 1: (Exponential Stabilization): The problem of exponential stabilization consists of finding the controller parameters $\xi=\left(\xi_{1}^{\top}, \xi_{2}^{\top}, \xi_{3}^{\top}\right)^{\top}$ such that the eigenvalues of the Jacobian matrix $A(\xi):=\frac{\partial P}{\partial x}\left(x_{1}^{\star}, \xi\right)$ lie inside the unit circle.

\section{B. Iterative BMI Algorithm}

Using the Schur complement lemma, it can be shown that Problem 1 is equivalent to the existence of $W=W^{\top}>$ 0 and $\gamma \in(0,1)$ such that the following nonlinear matrix inequality (NMI) is satisfied

$$
\left[\begin{array}{cc}
W & A(\xi) W \\
\star & (1-\gamma) W
\end{array}\right]>0 .
$$

In particular, $V(\delta x):=\delta x^{\top} W^{-1} \delta x$ is a Lyapunov candidate for (16) such that $V[k+1]-V[k]<-\gamma V[k]$. Here, $1-\gamma$ is an upper bound on the spectral radius of the Jacobian matrix $A(\xi)$ to tune the convergence rate of solutions of the discrete-time system (15) to the fixed point $x_{1}^{\star}$. There is no closed-form expression for the high-dimensional Jacobian matrix $A(\xi)$, which complicates the design of the controller parameters $\xi$. In order to find a stabilizing set of controller parameters $\xi$ satisfying the NMI (17), we employ the iterative BMI algorithm developed in [37]. The BMI algorithm designs a sequence of controller parameters $\left\{\xi^{\ell}\right\}$, where the superscript $\ell \in\{0,1,2, \cdots\}$ represents the iteration number. The objective is to converge to a set of parameters $\xi^{\ell}$ that solve Problem 1 or equivalently (17). The algorithm has the following three steps.

Step 1 (Sensitivity Analysis): During the iteration number $\ell$, we approximate the Jacobian matrix $A\left(\xi^{\ell}+\Delta \xi\right)$ with its first-order Taylor series expansion, that is,

$$
\begin{aligned}
A\left(\xi^{\ell}+\Delta \xi\right) & \approx A\left(\xi^{\ell}\right)+\bar{A}\left(\xi^{\ell}\right)(I \otimes \Delta \xi) \\
& =: \hat{A}\left(\xi^{\ell}, \Delta \xi\right),
\end{aligned}
$$

\footnotetext{
${ }^{1}$ Since $\operatorname{dim}\left(\hat{\mathcal{S}}_{1 \rightarrow 2}\right)=\operatorname{dim}\left(\mathcal{X}_{1}\right)-1=2\left(n_{q}-3\right)-1$, the Jacobian matrix $\frac{\partial P}{\partial x}\left(x_{1}^{\star}, \xi\right)$ is indeed an $\left(2 n_{q}-7\right) \times\left(2 n_{q}-7\right)$ matrix that maps the tangent space $\mathrm{T}_{x_{1}^{\star}} \hat{\mathcal{S}}_{1 \rightarrow 2}$ back to itself.
} 
where " $\otimes$ " represents the Kronecker product and $\Delta \xi$ is a sufficiently small increment in controller parameters. An effective numerical approach to compute the sensitivity matrix $\bar{A}\left(\xi^{\ell}\right)$ based on the variational equation has been developed in [30, Theorem 2]. The objective of the sensitivity analysis is to replace the nonlinear matrix $A\left(\xi^{\ell}+\Delta \xi\right)$ with its firstorder approximation $\hat{A}\left(\xi^{\ell}, \Delta \xi\right)$ that is an affine function in terms of $\Delta \xi$. This will reduce the NMI of (17) into a BMI in Step 2 that can be effectively solved with available software packages such as PENBMI [47].

Step 2 (BMI Optimization Problem): The objective of the BMI optimization problem is to solve for the increment in controller parameters $\Delta \xi$ such that the eigenvalues of the approximated Jacobian matrix $\hat{A}\left(\xi^{\ell}, \Delta \xi\right)$ lie inside the unit circle. In particular, we setup the following BMI optimization problem:

$$
\begin{array}{ll}
\min _{(W, \Delta \xi, \eta, \gamma)} & -w \gamma+\eta \\
\text { s.t. } \quad & {\left[\begin{array}{cc}
W & \hat{A}\left(\xi^{\ell}, \Delta \xi\right) W \\
\star & (1-\gamma) W
\end{array}\right]>0} \\
& {\left[\begin{array}{cc}
I & \Delta \xi \\
\star & \eta
\end{array}\right]>0} \\
& \gamma>0
\end{array}
$$

where (20) is a BMI condition, and from the LMI (21) and the Schur complement lemma, $\eta$ is an upper bound on the 2-norm of $\Delta \xi$, i.e., $\eta>\|\Delta \xi\|_{2}^{2}$. The cost function (19) then minimizes a weighted sum of $-\gamma$ and $\mu$ with the weighting factor $w>0$ as a tradeoff between improving the convergence rate and minimizing $\eta$ to have a good approximation based on the Taylor series expansion.

Step 3 (Iteration): Let us assume that $\left(W^{\ell \star}, \Delta \xi^{\ell \star}, \eta^{\ell \star}, \gamma^{\ell \star}\right)$ represent a local minimum (not necessarily a global solution) for the BMI optimization problem (19)-(22). Then, Step 3 updates the controller parameters for the next iteration as

$$
\xi^{\ell+1}=\xi^{\ell}+\Delta \xi^{\ell \star}
$$

If the requirements of Problem 1 are satisfied at $\xi=\xi^{\ell+1}$, the algorithm is successful and stops. Otherwise, it continues by coming back to Step 1 (sensitivity analysis around $\xi^{\ell+1}$ ). If the BMI optimization problem in Step 2 is not feasible, the algorithm is not successful and stops.

\section{Convergence of the Iterative BMI Algorithm}

Our previous work in [37] presented a set of sufficient conditions to guarantee the convergence of the iterative algorithm to a stabilizing solution. These conditions were developed based on the third-order derivative of the Poincare map and require extensive computational burden. This section however presents an alternative set of sufficient conditions for the finite-time convergence of the algorithm that can be effectively verified. The new conditions are based on the second-order derivative of the Poincaré map. In particular, we assume a convex-hull of the approximate Jacobian matrices during the previous iterations and if the current real Jacobian matrix takes values in this convex-hull, the algorithm successfully converges. To make this statement clear, we present the following theorem.

Theorem 1 (Finite Time Convergence): Assume that Assumptions 1-3 are satisfied. Suppose that the BMI optimization problem (19)-(22) is feasible during $N$ iterations for some positive integer $N$. Let $\left\{\xi^{\ell}\right\}_{\ell=0}^{N}$ denote the generated sequence of controller parameters from the initial guess $\xi^{0}$, i.e., $\xi^{\ell}=\xi^{0}+\sum_{j=0}^{\ell-1} \Delta \xi^{j \star}, \ell \in\{1,2, \cdots, N\}$, and $\hat{A}^{\ell}:=\hat{A}\left(\xi^{\ell}, \Delta \xi^{\ell \star}\right)$ represent the approximated Jacobian matrix during the iteration $\ell \in\{0,1, \cdots, N-1\}$. Define the following convex-hull of approximated Jacobian matrices

$\mathcal{A}:=\left\{\sum_{\ell=0}^{N-1} \alpha_{\ell} \hat{A}^{\ell} \mid \alpha_{\ell} \geq 0, \ell=0, \cdots, N-1, \sum_{\ell=0}^{N-1} \alpha_{\ell}=1\right\}$. If $A\left(\xi^{N}\right) \in \mathcal{A}$, then $\xi=\xi^{N}$ forms a set of stabilizing controller parameters satisfying Problem 1.

Proof: From the feasibility of the BMI optimization problem (19)-(22), $V(\delta x)=\delta x^{\top}\left(W^{\ell \star}\right)^{-1} \delta x$ is a Lyapunov function for the discrete-time system $\delta x[k+1]=\hat{A}^{\ell} \delta x[k]$. Since $A\left(\xi^{N}\right)=\sum_{\ell=0}^{N-1} \alpha_{\ell} \hat{A}^{\ell}$, from [48, Theorem 2], one can conclude that $V(\delta x)=\delta x^{\top} Q \delta x$ is Lyapunov function for $\delta x[k+1]=A\left(\xi^{N}\right) \delta x[k]$, where $Q:=$ $\sum_{\ell=0}^{N-1} \alpha_{\ell}\left(W^{\ell \star}\right)^{-1}$. This completes the exponential stability of the periodic orbit $\mathcal{O}$ as stated in Problem 1.

\section{NUMERICAL ANALYSIS AND SIMULATIONS}

This section employs the iterative BMI algorithm to look for virtual constraints that exponentially stabilize walking gaits for a multi-domain hybrid model of a 3D bipedal robot.

\section{A. 3D Robot Model}

The robot model consists of a rigid tree structure with a torso link and two identical legs terminating at nontrivial feet (see Fig. 2). Each leg of the robot includes 4 actuated joints: a 2 DOF hip (ball) joint with roll and pitch motions, a 1 DOF knee joint in the sagittal plane, plus a 1 DOF ankle joint in the sagittal plane (no ankle joint in the frontal plane). During the flight phase, the mechanical system has $n_{q}=14$ DOFs with $m=8$ actuators. To describe the configuration variables, we rigidly attach a frame to the torso link with the $z$-axis being upward and the $y$-axis being in the direction of walking. Three position variables are assigned to describe the translational motion of the torso frame with respect to a world frame. The orientation of the torso frame with respect to the ground is then described by three Euler angles, referred to as the yaw, roll, and pitch angles. The remaining eight configuration variables are assigned to the actuated joints of the legs. The mechanical system has 3 and 1 degrees of underactuation during Phases 1 and 3, respectively. The kinematic and dynamic parameter values for the links are taken according to those reported in [49] from a human cadaver study. A desired periodic gait $\mathcal{O}$ is designed using the motion planning algorithm of [14] for walking at 0.8 $(\mathrm{m} / \mathrm{s})$. The controller parameters to be determined include the columns of the output matrices $H_{1} \in \mathbb{R}^{8 \times 14}, H_{2} \in$ 
$\mathbb{R}^{7 \times 14}$, and $H_{3} \in \mathbb{R}^{8 \times 14}$ together with the columns of the gain matrix $K_{2} \in \mathbb{R}^{1 \times 28}$. However, we set the columns corresponding to the translational motions to zero and just look for the columns corresponding to the torso Euler angles and internal shape variables. In particular, we solve for 275 (i.e., $(8+7+8) \times 11+22)$ parameters, i.e., $\xi \in \mathbb{R}^{275}$. It can be shown that using this assumption, the state feedback laws $\Gamma_{i}\left(x, \xi_{i}\right)$ do not depend on the translational position and velocity measurements.

\section{B. PENBMI Solver}

BMIs are NP-hard problems. However, PENBMI is a general purpose and local solver which guarantees the convergence to a critical point satisfying the Karush-KuhnTucker optimality conditions [47]. We start with an initial guess $\xi^{0}$ for which the controlled variables, i.e., $H_{i} q$ for $i \in$ $\mathcal{I}$, only include the shape variables. The controller gain $K_{2}$ embedded in $\xi^{0}$ is also set to zero. For this set of controller parameters, the spectral radius of the $21 \times 21$ Jacobian matrix $A\left(\xi^{0}\right)$ becomes 4.7536 , and hence, the gait is unstable. To exponentially stabilize the gait, we employ the iterative BMI algorithm, in which the BMI optimization problem is solved with PENBMI solver from TOMLAB [50] integrated with MATLAB environment through YALMIP [51]. The BMI optimization procedure on a computer with a dual 2.3 GHz Intel Xeon E5-2670 v3 processor takes approximately 20 minutes. After 8 iterations, the algorithm successfully converges to a stabilizing feedback control solution for which the spectral radius of the Jacobian matrix becomes 0.8572 . Figure 2 depicts the phase portraits of the BMI optimized closed-loop system. Here, the simulation starts off of the orbit at the beginning of the right stance phase. Convergence to the periodic orbit even in the yaw component is clear. The animation of this simulation together with the optimal controller parameters can be found at [52].

\section{CONCLUSIONS}

This paper presented an algorithm to systematically design continuous-time controllers that exponentially stabilize periodic gaits for multi-domain hybrid models of bipedal walking. The algorithm considers a general form of parameterized and nonlinear feedback controllers for different phases of the walking cycle. The exponential stabilization problem and Poincaré map are investigated to extend the BMI optimization approach for the stabilization of multidomain hybrid periodic orbits. The algorithm is based on an iterative sequence of optimization problems involving BMIs and LMIs. A new set of sufficient conditions are presented to guarantee the convergence of the algorithm to a stabilizing solution at a finite number of iterations. The conditions can be effectively verified and require less computational effort compared to those of [37]. The numerical results illustrate the power of the algorithm in designing stabilizing virtual constraint controllers for dynamic walking of a 3D human model with 28 state variables and 275 control parameters. For future research, we will investigate the scalability and capability of the algorithm in stabilizing periodic orbits
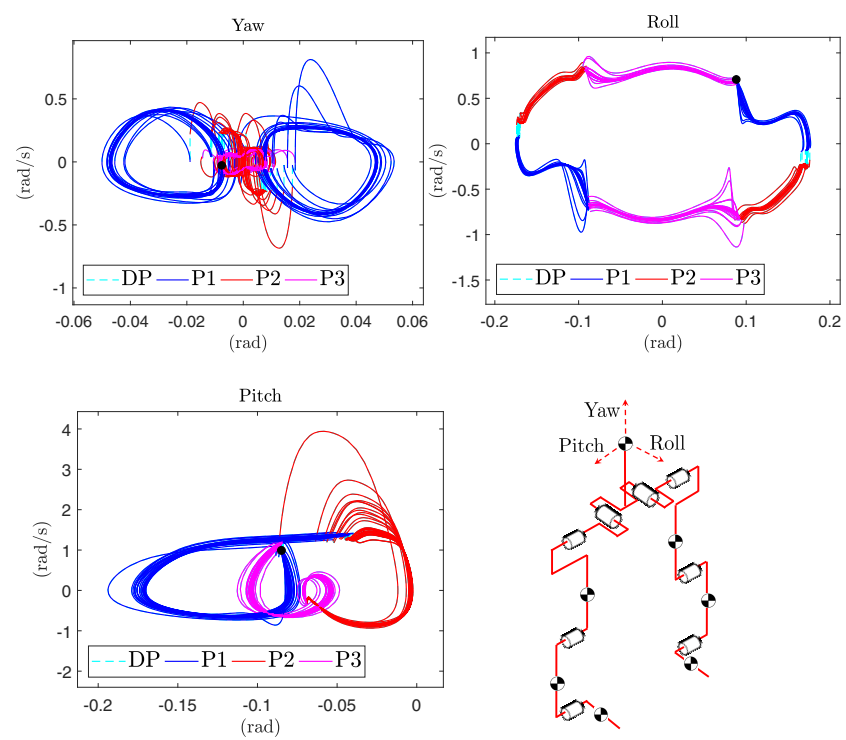

Fig. 2: Phase portraits for the torso Euler angles (yaw, roll and pitch) during 100 consecutive steps of 3D anthropomorphic walking with the BMI optimized feedback controllers together with the structure of the 14 DOFs bipedal robot. "DP", "P1", "P2", and "P3" stand for the discrete-time phases, Phase 1, Phase 2, and Phase 3, respectively.

for multi-contact bipedal walking gaits with frontal ankle joints. We will also study the capability of the algorithm in designing $\mathcal{H}_{2}$ and $\mathcal{H}_{\infty}$ controllers for walking on rough terrain [53]. An alternative future direction will be extending the work of [37] to design decentralized nonlinear feedback controllers for anthropomorphic multi-contact walking with application to powered prosthetic legs.

\section{REFERENCES}

[1] A. Goswami, "Postural Stability of Biped Robots and the FootRotation Indicator (FRI) Point," The International Journal of Robotics Research, vol. 18, no. 6, pp. 523-533, 1999.

[2] M. Vukobratović, B. Borovac, and D. Surla, Dynamics of Biped Locomotion. Springer, 1990.

[3] M. Spong and F. Bullo, "Controlled symmetries and passive walking," Automatic Control, IEEE Transactions on, vol. 50, no. 7, pp. 10251031, July 2005.

[4] A. Ames and S. Sastry, "Hybrid geometric reduction of hybrid systems," in Decision and Control, 45th IEEE Conference on, Dec 2006, pp. 923-929.

[5] A. Ames, R. Gregg, and M. Spong, "A geometric approach to threedimensional hipped bipedal robotic walking," in Decision and Control, 46th IEEE Conference on, Dec 2007, pp. 5123-5130.

[6] I. Manchester, U. Mettin, F. Iida, and R. Tedrake, "Stable dynamic walking over uneven terrain," The International Journal of Robotics Research, vol. 30, no. 3, pp. 265-279, 2011.

[7] E. Westervelt, J. Grizzle, and D. Koditschek, "Hybrid zero dynamics of planar biped walkers," Automatic Control, IEEE Transactions on, vol. 48, no. 1, pp. 42-56, Jan 2003.

[8] B. Morris and J. Grizzle, "Hybrid invariant manifolds in systems with impulse effects with application to periodic locomotion in bipedal robots," Automatic Control, IEEE Transactions on, vol. 54, no. 8, pp. 1751-1764, Aug 2009.

[9] R. Gregg and L. Righetti, "Controlled reduction with unactuated cyclic variables: Application to 3D bipedal walking with passive yaw rotation," Automatic Control, IEEE Transactions on, vol. 58, no. 10, pp. 2679-2685, Oct 2013. 
[10] C. Chevallereau, G. Abba, Y. Aoustin, F. Plestan, E. Westervelt, C. Canudas-de Wit, and J. Grizzle, "RABBIT: a testbed for advanced control theory," Control Systems Magazine, IEEE, vol. 23, no. 5, pp. 57-79, Oct 2003.

[11] K. Sreenath, H.-W. Park, I. Poulakakis, and J. Grizzle, "Embedding active force control within the compliant hybrid zero dynamics to achieve stable, fast running on MABEL," The International Journal of Robotics Research, vol. 32, no. 3, pp. 324-345, 2013.

[12] A. E. Martin, D. C. Post, and J. P. Schmiedeler, "The effects of foot geometric properties on the gait of planar bipeds walking under HZD-based control," The International Journal of Robotics Research, vol. 33, no. 12, pp. 1530-1543, 2014.

[13] J. Lack, M. Powell, and A. Ames, "Planar multi-contact bipedal walking using hybrid zero dynamics," in Robotics and Automation, IEEE International Conference on, May 2014, pp. 2582-2588.

[14] A. Ramezani, J. Hurst, K. Akbai Hamed, and J. Grizzle, "Performance analysis and feedback control of ATRIAS, a three-dimensional bipedal robot," Journal of Dynamic Systems, Measurement, and Control December, ASME, vol. 136, no. 2, December 2013.

[15] A. Hereid, E. A. Cousineau, C. M. Hubicki, and A. D. Ames, "3D dynamic walking with underactuated humanoid robots: A direct collocation framework for optimizing hybrid zero dynamics," in 2016 IEEE International Conference on Robotics and Automation (ICRA), May 2016, pp. 1447-1454.

[16] H.-W. Park, A. Ramezani, and J. Grizzle, "A finite-state machine for accommodating unexpected large ground-height variations in bipedal robot walking," Robotics, IEEE Transactions on, vol. 29, no. 2, pp. 331-345, April 2013.

[17] C. O. Saglam and K. Byl, "Meshing hybrid zero dynamics for rough terrain walking," in 2015 IEEE International Conference on Robotics and Automation (ICRA), May 2015, pp. 5718-5725.

[18] J. P. Reher, A. Hereid, S. Kolathaya, C. M. Hubicki, and A. D. Ames, "Algorithmic foundations of realizing multi-contact locomotion on the humanoid robot DURUS," in Twelfth International Workshop on Algorithmic Foundations on Robotics, 2016.

[19] H. Zhao, A. Hereid, W.-1. Ma, and A. D. Ames, "Multi-contact bipedal robotic locomotion," Robotica, pp. 1-35, 2015.

[20] R. Gregg, T. Lenzi, L. Hargrove, and J. Sensinger, "Virtual constraint control of a powered prosthetic leg: From simulation to experiments with transfemoral amputees," Robotics, IEEE Transactions on, vol. 30, no. 6, pp. 1455-1471, Dec 2014.

[21] R. Gregg and J. Sensinger, "Towards biomimetic virtual constraint control of a powered prosthetic leg," Control Systems Technology, IEEE Transactions on, vol. 22, no. 1, pp. 246-254, Jan 2014.

[22] A. Martin and R. Gregg, "Hybrid invariance and stability of a feedback linearizing controller for powered prostheses," in American Control Conference, 2015, pp. 4670-4676.

[23] H. Zhao, J. Horn, J. Reher, V. Paredes, and A. Ames, "Multicontact locomotion on transfemoral prostheses via hybrid system models and optimization-based control," IEEE Transactions on Automation Science and Engineering, vol. 13, no. 2, pp. 502-513, April 2016.

[24] H. Zhao, E. Ambrose, and A. D. Ames, "Preliminary results on energy efficient $3 \mathrm{D}$ prosthetic walking with a powered compliant transfemoral prosthesis," in Robotics and Automation (ICRA), 2017 IEEE International Conference on. IEEE, 2017, pp. 1140-1147.

[25] H. Zhao, A. Hereid, E. Ambrose, and A. D. Ames, "3D multi-contact gait design for prostheses: Hybrid system models, virtual constraints and two-step direct collocation," in Decision and Control (CDC), 2016 IEEE 55th Conference on. IEEE, 2016, pp. 3668-3674.

[26] A. Agrawal, O. Harib, A. Hereid, S. Finet, M. Masselin, L. Praly, A. Ames, K. Sreenath, and J. Grizzle, "First steps towards translating hzd control of bipedal robots to decentralized control of exoskeletons," IEEE Access, vol. 5, pp. 9919-9934, 2017.

[27] I. Poulakakis and J. Grizzle, "The spring loaded inverted pendulum as the hybrid zero dynamics of an asymmetric hopper," Automatic Control, IEEE Transactions on, vol. 54, no. 8, pp. 1779-1793, Aug 2009.

[28] Q. Cao and I. Poulakakis, "Quadrupedal running with a flexible torso: control and speed transitions with sums-of-squares verification," Artificial Life and Robotics, vol. 21, no. 4, pp. 384-392, Dec 2016.

[29] A. Isidori, Nonlinear Control Systems. Springer; 3rd edition, 1995.

[30] K. Akbari Hamed, B. Buss, and J. Grizzle, "Exponentially stabilizing continuous-time controllers for periodic orbits of hybrid systems: Application to bipedal locomotion with ground height variations," The
International Journal of Robotics Research, vol. 35, no. 8, pp. 977999, 2016.

[31] C. Chevallereau, J. Grizzle, and C.-L. Shih, "Asymptotically Stable Walking of a Five-Link Underactuated 3-D Bipedal Robot," Robotics, IEEE Transactions on, vol. 25, no. 1, pp. 37-50, Feb 2009.

[32] E. Westervelt, J. Grizzle, C. Chevallereau, J. Choi, and B. Morris, Feedback Control of Dynamic Bipedal Robot Locomotion. Taylor \& Francis/CRC, 2007.

[33] J. Grizzle, G. Abba, and F. Plestan, "Asymptotically stable walking for biped robots: analysis via systems with impulse effects," Automatic Control, IEEE Transactions on, vol. 46, no. 1, pp. 51-64, Jan 2001.

[34] W. Haddad, V. Chellaboina, and S. Nersesov, Impulsive and Hybrid Dynamical Systems: Stability, Dissipativity, and Control. Princeton University Press, July 2006.

[35] T. Parker and L. Chua, Practical Numerical Algorithms for Chaotic Systems. Springer, 1989.

[36] K. Akbari Hamed and J. Grizzle, "Event-based stabilization of periodic orbits for underactuated 3-D bipedal robots with left-right symmetry," Robotics, IEEE Transactions on, vol. 30, no. 2, pp. 365-381, April 2014.

[37] K. Akbari Hamed and R. D. Gregg, "Decentralized feedback controllers for robust stabilization of periodic orbits of hybrid systems: Application to bipedal walking," Control Systems Technology, IEEE Transactions on, vol. 25, no. 4, pp. 1153-1167, July 2017.

[38] K. Akbari Hamed and J. W. Grizzle, "Reduced-order framework for exponential stabilization of periodic orbits on parameterized hybrid zero dynamics manifolds: Application to bipedal locomotion," Nonlinear Analysis: Hybrid Systems, vol. 25, pp. 227-245, August 2017.

[39] B. Buss, K. Akbari Hamed, B. A. Griffin, and J. W. Grizzle, "Experimental results for 3D bipedal robot walking based on systematic optimization of virtual constraints," in 2016 American Control Conference (ACC), July 2016, pp. 4785-4792.

[40] Dynamic Leg Locomotion YouTube Channel. (2015) MARLO: Dynamic 3D walking based on HZD gait design and BMI constraint selection, https://www.youtube.com/watch? $\mathrm{v}=5 \mathrm{~ms} 5 \mathrm{DtPNwHo}$.

[41] A. D. Ames, R. Vasudevan, and R. Bajcsy, "Human-data based cost of bipedal robotic walking," in Proceedings of the 14th international conference on Hybrid systems: computation and control. ACM, 2011, pp. $153-162$.

[42] J. W. Grizzle, C. Chevallereau, R. W. Sinnet, and A. D. Ames, "Models, feedback control, and open problems of 3D bipedal robotic walking," Automatica, vol. 50, no. 8, pp. 1955-1988, 2014.

[43] Y. Hurmuzlu and D. B. Marghitu, "Rigid body collisions of planar kinematic chains with multiple contact points," vol. 13, no. 1, pp. 82-92, 1994.

[44] S. Burden, S. Revzen, and S. Sastry, "Model reduction near periodic orbits of hybrid dynamical systems," Automatic Control, IEEE Transactions on, vol. 60, no. 10, pp. 2626-2639, Oct 2015.

[45] M. Maggiore and L. Consolini, "Virtual holonomic constraints for Euler Lagrange systems," Automatic Control, IEEE Transactions on, vol. 58, no. 4, pp. 1001-1008, April 2013.

[46] A. Shiriaev, A. Sandberg, and C. Canudas de Wit, "Motion planning and feedback stabilization of periodic orbits for an acrobot," in Decision and Control. 43rd IEEE Conference on, vol. 1, Dec 2004, pp. 290-295 Vol.1.

[47] D. Henrion, J. Lofberg, M. Kocvara, and M. Stingl, "Solving polynomial static output feedback problems with PENBMI," in Decision and Control, and European Control Conference. 44th IEEE Conference on, Dec 2005, pp. 7581-7586.

[48] M. de Oliveira, J. Bernussou, and J. Geromel, "A new discrete-time robust stability condition," Systems \& Control Letters, vol. 37, no. 4, pp. 261-265, 1999.

[49] P. de Leva, "Adjustments to Zatsiorsky-Seluyanov's segment inertia parameters," J Biomech, vol. 29, no. 9, pp. 123-1230, 1996.

[50] TOMLAB optimization, http://tomopt.com/tomlab/.

[51] J. Lofberg, "YALMIP: a toolbox for modeling and optimization in MATLAB," in Computer Aided Control Systems Design, 2004 IEEE International Symposium on, Sept 2004, pp. 284-289.

[52] (2017) Exponentially Stabilizing Controllers for Multi-Contact 3D Bipedal Locomotion, https://youtu.be/nem 1 kbltg4U.

[53] H. Dai and R. Tedrake, " $\mathcal{L}_{2}$-gain optimization for robust bipedal walking on unknown terrain," in Robotics and Automation, IEEE International Conference on, May 2013, pp. 3116-3123. 